\title{
Medium-term adoption trends for laparoscopic, robotic and transanal total mesorectal excision (TaTME) techniques
}

\author{
G. Pellino $0^{1,2} \cdot$ J. Warusavitarne $e^{1,3}$
}

Received: 14 October 2017 / Accepted: 17 October 2017 / Published online: 16 November 2017

๑) Springer International Publishing AG, part of Springer Nature 2017

Laparoscopic colorectal surgery (LCS) was first described in the 1990s and for the most part has become the standard of care for colorectal surgery. Robotic-assisted colorectal surgery (RACS) was described around the year 2000, and advocates argue that it overcomes many issues associated with laparoscopic surgery, particularly in surgery for rectal cancer. More recently, the bottom-up minimally invasive approach, transanal total mesorectal excision (TaTME), for rectal surgery has gained popularity, and it has perceived advantages over laparoscopic rectal surgery.

Minimally invasive surgery has been associated with some short-term benefits, including reduced bleeding and postoperative pain, and better cosmetic outcomes. Despite this, the debate on oncologic outcomes when compared with open surgery is still ongoing [1-3]. The benefits of one minimally invasive approach over the others form the subject of further debate, and as much of this is driven by proponents of an individual technique, choosing the most appropriate technique can be difficult $[1,2]$. Despite the enthusiasm of the proponents, many other factors play a part in the choice of technique and these can be broadly described as those related to the patient, surgeon and individual hospital or a country.

We compared LCS, RACS and TaTME/TAMIS using the following criteria: (1) number of studies published in three time frames $(I=$ within the first 2 years after acceptance of the technique; $\mathrm{II}=$ during the subsequent 3 years;

J. Warusavitarne

j.warusavitarne@nhs.net

1 St Mark's Hospital and Academic Institute, Imperial College University of London, Northwick Park, Watford Road, Harrow, Middlesex HA13UJ, UK

2 Department of Medical, Surgical, Neurological, Metabolic and Ageing Sciences, University of Campania "Luigi Vanvitelli’, Naples, Italy

3 Department of Surgery and Cancer, Imperial College, London, UK
III = 3 years after II, if available); (2) number of involved patients; (3) predicted/actual trends. We searched PubMed and only included randomized controlled trials, clinical trials, observational, case-control and cohort studies (time frames: laparoscopy-I: 1993-1994, II: 1995-1997, III: 1997-2000; robotically assisted-I: Dec 2004-2006, II: 2007-2009, III: 2010-2012; transanal proctectomy-I: 2013-2014, II: 2015-2016). The findings are summarized in Figs. 1 and 2. In the early years, the USA contributed majority of publications related to LCS, with China contributing at the lower end of the spectrum. By the II time frame, there were more contributions from Western Europe, Japan and Australia. In the III time frame, there was a notable increase in reporting on LCS with more contributions from the USA and Western Europe as well as the UK and Scandinavian countries, in addition to Japan, Australia and China. For RACS, in the early time period there were a few manuscripts from the USA and Europe. During the subsequent time frame, reports from the USA continued to increase, whereas there was less reporting from European countries with the exception of the UK. There was also more reporting of robotic surgery from South Korea, while the enthusiasm from Europe was not as strong as that for LCS. The USA and South Korea appear to be leading the charge in this regard. This begs the question whether this is mainly related to the cost of the procedure. The concept of TaTME originated in the USA with some individuals promoting the technique within Europe. During the II time frame, adoption of TaTME mushroomed to include many continental European countries, while adoption of the technique was being introduced rapidly in the USA and UK. Figure 2 shows the trends of LCS, RACS and TaTME in the different time frames. LCS had a gradual but constant increase over time, with an expected further increase probably as experience was disseminated by training a new generation of surgeons. RACS growth was slower during the second time frame, but increased considerably during the third, with an almost threefold increase. TaTME trend sits between these two, in 


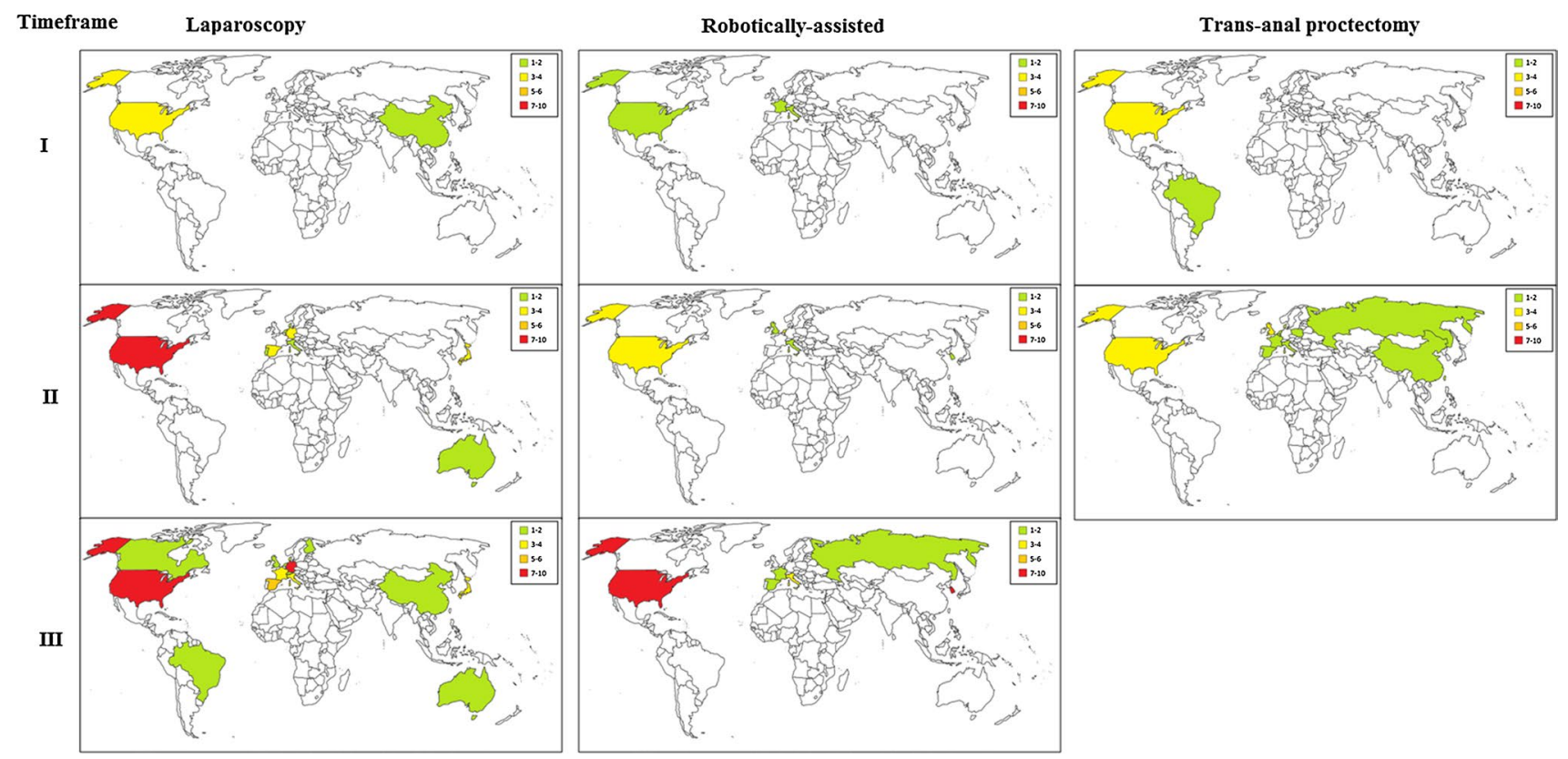

Fig. 1 Heat map of the number of publications over time in laparoscopic colorectal surgery, robotic-assisted colorectal surgery and transanal total mesorectal excision/transanal minimally invasive surgery

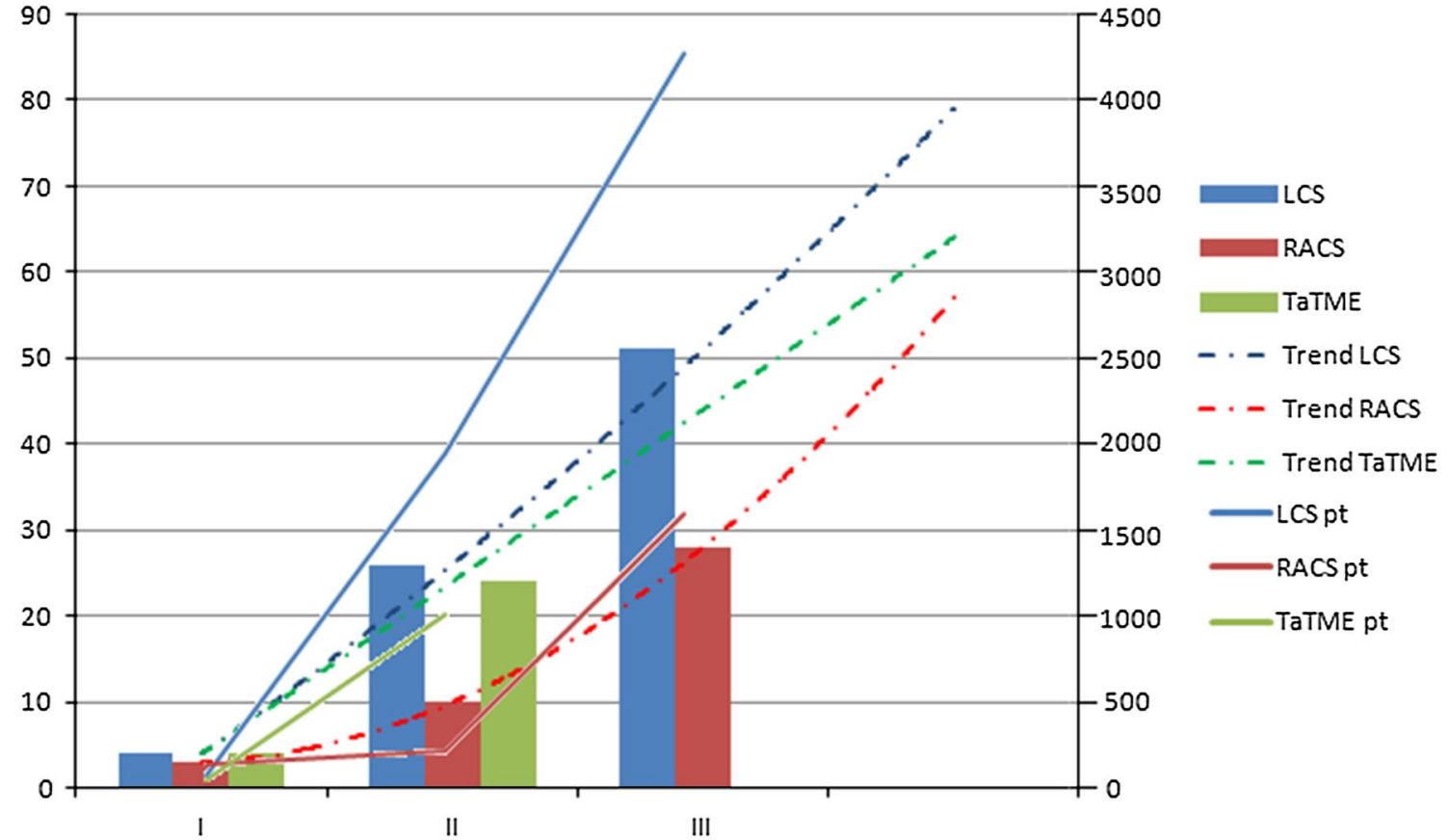

Fig. 2 Trend over time of publications (squares) with associated estimated projection (dotted lines) and enrolled patients (lines). Numbers on the left represent publications, numbers on the right represent patients. LCS laparoscopic colorectal surgery, RACS robotic-assisted colorectal surgery, TaTME transanal total mesorectal excision

procedure-related complications, outcomes or even insight into learning curves. The published literature does not always give an accurate reflection of the global adoption of the technique as centers with low-volume experience may 
not necessarily publish their experience. Notwithstanding these inherent issues, analyzing globally published data still give important insight into a particular procedure and its evolution. The number of patients undergoing LCS showed a steep increase over time, reaching almost 4500 in the last time frame. On the contrary, patients receiving RACS had a marginal increase between I and II period, reflecting the plateau reached in the different centers that might be justified by the high costs and long operative time associated with the procedure. Where RACS is concerned, there was a reasonable increase in the number of patients treated over time frame III, but probably the costs associated with the procedure have not allowed the growth seen in LCS. In contrast, there has been a marked increase in TaTME procedures, and considering that this technique is only applicable to rectal diseases, this growth can only be interpreted as quite considerable. This concept can be further supported by the fact that national training programs are being developed to ensure that there is safe introduction of TaTME across Europe.

One of the most important factors that must be considered in novel technologies is the training required to master the procedure. We suggest that this goes beyond the "learning curve," specific to each device and procedure, which is desirable and represents a valid tool. Besides the overall number of procedures performed, other important parameters need to be assessed. Foster et al. [4] have recently proposed an objective clinical human reliability analysis (OCHRA) to assess the proficiency of surgeons performing LCS via video recording. Even if promising, it has been suggested that it requires further validation and improvement. TaTME pioneers faced a high number of complications in their early experience, which was mainly due to the learning curve associated with adopting a new technique. On this basis, a robust pilot training program was implemented and this included teaching, video assessment, cadaver courses and assessment by a proctor [5]. Those who successfully complete the training are deemed safe to perform the procedure, but the learning curve is yet to be determined. Where RACS is concerned, the current certification requires simulator training, dry and wet laboratory experience as well as proctoring (https://www.sages.org/publications/guidelines/ consensus-document-robotic-surgery/).

In addition to training and certification, early adopters of TaTME established an international registry (http://www. lorec.nhs.uk/system/content.asp?pkey=23). The use of prospective registries should be encouraged to monitor the safety and effectiveness of new procedures. For TaTME, it fostered the ability to tackle the issues identified by analyzing data. In this perspective, TaTME advocates have mirrored LCS for which an international registry was commenced in 1993.
Whichever the technique used, it is important that it is carried out in high-volume centers, as surgeons with lower caseload are more prone to have postoperative complications. Keller et al. [6] suggested that lower-volume providers were performing the majority of RACS in the USA, resulting in increased complications.

While the debate over the quality of specimen and oncological result between the three techniques is still on, there appears to be no compelling reason for preferring one technique over the other in terms of short-term outcomes.

It is likely that these techniques are not really competitors, but rather complementary. Ultimately, it is outcomes that matter, and outcomes have been shown to be highly operator dependent. Therefore, the more likely scenario is that surgeon preference and technique together with budgetary constraints will determine the best minimally invasive approach rather than one being more superior to the other.

Funding The authors did not receive any funding for this paper.

\section{Compliance with ethical standards}

Conflict of interest The authors declare that they have no conflict of interest.

Ethical approval The present paper was exempt from approval.

Informed consent For this review of literature, informed consent was not required.

\section{References}

1. Abbas SK, Yelika SB, You K, Mathai J, Essani R, Krivokapić Z, Bergamaschi R (2017) Rectal cancer should not be resected laparoscopically: the rationale and the data. Tech Coloproctol 21:237-240. https://doi.org/10.1007/s10151-017-1596-x

2. Shawki S, Liska D, Delaney CP (2017) Laparoscopic surgery for rectal cancer: the verdict is not final yet! Tech Coloproctol 21:241-243. https://doi.org/10.1007/s10151-017-1594-z

3. Martínez-Pérez A, Carra MC, Brunetti F, de 'Angelis N (2017) Pathologic outcomes of laparoscopic vs open mesorectal excision for rectal cancer: a systematic review and meta-analysis. JAMA Surg 152:e165665. https://doi.org/10.1001/jamasurg.2016.5665

4. Foster JD, Miskovic D, Allison AS, Conti JA, Ockrim J, Cooper EJ, Hanna GB, Francis NK (2016) Application of objective clinical human reliability analysis (OCHRA) in assessment of technical performance in laparoscopic rectal cancer surgery. Tech Coloproctol 20:361-367. https://doi.org/10.1007/s10151-016-1444-4

5. Penna M, Hompes R, Mackenzie H, Carter F, Francis NK (2016) First international training and assessment consensus workshop on transanal total mesorectal excision (taTME). Tech Coloproctol 20:343-352

6. Keller DS, Hashemi L, Lu M, Delaney CP (2013) Short-term outcomes for robotic colorectal surgery by provider volume. J Am Coll Surg 217(1063-9):e1. https://doi.org/10.1016/j. jamcollsurg.2013.07.390 